Théologiques

Théologiques

\title{
La théologie dans l'université : un défi à la théologie. Quelques remarques
}

\section{Jean-Claude Petit}

Volume 1, numéro 1, mars 1993

Théologie et sciences humaines en contexte universitaire

URI : https://id.erudit.org/iderudit/602380ar

DOI : https://doi.org/10.7202/602380ar

Aller au sommaire du numéro

\section{Éditeur(s)}

Faculté de théologie de l'Université de Montréal

\section{ISSN}

1188-7109 (imprimé)

1492-1413 (numérique)

Découvrir la revue

\section{Citer cet article}

Petit, J.-C. (1993). La théologie dans l'université : un défi à la théologie. Quelques remarques. Théologiques, 1(1), 11-31.

https://doi.org/10.7202/602380ar
Résumé de l'article

Dans son sens technique, la théologie naît en Occident comme discipline académique. Exigence de la foi, elle ne peut se déployer qu'au coeur des possibilités de son époque et en dialogue avec la pensée de son temps. Cette exigence a souvent été perçue par des croyants comme une inacceptable manoeuvre pour diluer le vin fort de la foi. Elle seule peut cependant faire en sorte que la foi ne dégénère pas en gnose. C'est en s'inscrivant comme discipline herméneutique parmi les autres sciences de l'homme que la théologie peut aujourd'hui accomplir sa tâche, consciente d'être de part en part traversée par l'historicité qui marque toute pensée humaine. 


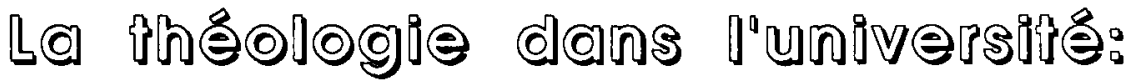 un ㅁำ
}

\section{Quelques remarques.}

\author{
Jean-Claude PETIT \\ Faculté de théologie \\ Université de Montréal
}

\section{RÉSUMÉ}

Dans son sens technique, la théologie naît en Occident comme discipline académique. Exigence de la foi, elle ne peut se déployer qu'au coeur des possibilités de son époque et en dialogue avec la pensée de son temps. Cette exigence a souvent été perçue par des croyants comme une inacceptable manoeuvre pour diluer le vin fort de la foi. Elle seule peut cependant faire en sorte que la foi ne dégénère pas en gnose. C'est en s'inscrivant comme discipline herméneutique parmi les autres sciences de l'homme que la théologie peut aujourd'hui accomplir sa tâche, consciente d'être de part en part traversée par l'historicité qui marque toute pensée humaine.

"Comment s'imaginer pouvoir éclairer sérieusement des questions qu'on n'a pas soi-même posées et qui laissent intacte sa propre position?"

Georges Morel

"Quelle maladie que ce besoin de donner des réponses." Job, $16: 3$

Dans son sens technique, la théologie naît en Occident comme discipline académique. Dans son grand ouvrage sur l'histoire de la méthode scolastique, Martin Grabmann a pu insister à juste titre sur le rôle qu'a joué dans l'émergence de la théologie comme science le 
développement des écoles dans l'Europe du Xlle siècle ${ }^{1}$. Ceci n'a pas été le fruit du hasard. On comprendrait très mal l'inscription universitaire de la théologie médiévale si l'on ne voyait pas que la théologie comme l'université naissante sont alors toutes deux parties prenantes d'une des plus fécondes renaissances de la pensée occidentale, expressions d'une expérience commune des possibilités et des exigences de la raison à leur époque ${ }^{2}$. C'est cette appartenance à l'émergence de ce qu'on peut appeler une nouvelle raison, dont on perçoit les appels bien avant qu'on ait apprivoisé tout le corpus aristotélicien, ainsi qu'à la nouvelle expérience du monde qui la sous-tend, qui a finalement conduit à l'établissement de rapports extrêmement étroits entre la théologie et l'université.

II y a donc ainsi plus de 700 ans que la théologie occupe au sein de l'université une place importante. À l'Université de Montréal, la Faculté de théologie est une des trois facultés fondatrices, avec la Faculté de droit et la Faculté de médecine. Nous ne disposons d'aucune étude d'ensemble sur l'histoire de la théologie à l'université ${ }^{3}$. Les nombreuses études sectorielles nous rappellent cependant que les rapports qui se sont établis entre les deux ont toujours été des rapports tensionnels, des rapports qui ont parfois donné lieu à des affrontements vigoureux, qui ont souvent reposé sur des malentendus, voire sur une

1 M. Grabmann, Die Geschichte der scholastischen Methode. Nach den gedruckten und ungedruckten Quellen bearbeitet. 2. Band, Die scholastische Methode im 12. und beginnenden 13. Jahrhundert. Freiburg im Breisgau, Herderschen Verlagshandlung, 1911 , p. 9.

2 Cf. entre autres M. D. Chenu, La théologie au douzième siècle. Paris, J. Vrin, (Études de philosophie médiévale \#45), 2/1966 (1957), 413 p., en particulier pp. 19 51, et J. de Ghellinck, Le mouvement théologique du XIle siècle. Études, recherches et documents. Paris , J. Gabalda, 1914, 409 p. Du même aussi: L'essor de la littérature latine au XIIe siècle. Bruxelles-Bruges-Paris, DDB, (Museum Lessianum - Section historique \# 4-5), ${ }^{2} / 1955$ (1947), 584 p. G. Paré, A. Brunet, P. Tremblay, La Renaissance du XIIe siècle. Les écoles et l'enseignement. Paris, J. Vrin, 324 p. Voir aussi: A. Forest, F. von Steenberghen, M. de Gandillac, Le mouvement doctrinal du XIe au XIVe siècle. Paris, Bloud et Gay, (Histoire de l'Église depuis les origines jusqu'à nos jours \# 13), 1951, 5-176.

3 Sur l'histoire de la théologie comme discipline académique voir: J.C. Petit, La théologie. Sa nature, ses méthodes, son histoire, ses problèmes. Répertoire bibliographique international. Français. Allemand. Anglais. Montréal, Les Presses de l'Université de Montréal, 1979, 468 p. Sur la problématique Théologie/Université à notre époque: pp. 407-421. Pour la littérature depuis lors:voir la bibliographie à la fin du présent article (pp. 27-30). 
méconnaissance étonnante de ce qu'est la théologie, et qui ont régulièrement conduit la théologie à s'expliquer à nouveau.

Mon propos n'est pas du tout de fournir ici une quelconque contribution à l'histoire de ces deux institutions que sont, chacune à leur manière, la théologie et l'université, ni d'esquisser une nouvelle "apologia pro scientia mea". Beaucoup plus simplement, bien que d'une manière sans doute non moins risquée, je voudrais rappeler ce qui me paraît constituer aujourd'hui pour la théologie l'enjeu fondamental de son inscription universitaire. L'espace imparti et la complexité évidente de cette question ne permettront pas d'aller ici bien au-delà de quelques remarques. Inscrites au départ d'un projet audacieux et risqué d'une nouvelle revue théologique, elles peuvent au moins indiquer à quelle tâche une théologie actuelle s'estime conviée.

\section{Un projet contesté}

L'inscription universitaire de la théologie, entendons évidemment ici son engagement dans le projet de rationalité de l'uuniversitas scientiarum", n'a jamais fait l'unanimité, ni chez les autres composantes de l'université ni non plus chez les théologiens eux-mêmes. Le souvenir d'une origine heureuse, caractérisée par une belle unanimité et une totale convergence des intérêts et des investissements des divers intervenants, ne repose que sur un mythe qui habite souvent la pensée des origines et qui nous parle finalement bien plus des peurs ou des aspirations secrètes qui travaillent le présent qu'il ne nous renseigne sur ce qu'il en était alors. II n'est pas sans intérêt de nous rappeler que le projet théologique qui prend corps au Xle et au Xlle siècles et qui trouvera une figure paradigmatique dans la formulation qu'en proposera Thomas d'Aquin dans la deuxième moitié du XIlle siècle a toujours été vigoureusement contesté même parmi les théologiens. Albert le Grand, par exemple, le maître de Thomas, est poussé à une très vive réaction par ses détracteurs parmi ses propres collègues, ces “ignorants", écrit-il, "qui veulent combattre par tous les moyens l'usage de la philosophie, et surtout chez les Prêcheurs, où personne ne leur résiste, bêtes brutes qui blasphèment ce qu'ils ignorent" 4 .

4 Albert le Grand, In epistolas Dionysii Areop. Epist. VIII, n.2. Cité par M.D. Chenu, La théologie comme science au XIIle siècle. Paris, J. Vrin, (Bibliothèque Thomiste \# 33), 3/1969, p. 29. Aussi par E. Gilson, La philosophie au Moyen-Age. Des origines patristiques à la fin du XIVe siècle. Paris, Payot, (Bibliothèque 
Thomas lui-même devra faire face à une réaction non moins virulente. Elle est évidemment d'autant plus vive que le projet de Thomas va au coeur même de la question. Dans son commentaire du $D e$ Trinitate de Boèce, il en vient, en effet, à aborder la question de savoir comment dans la "sacra doctrina" peut se vérifier la notion de science. Son interprétation n'inaugure pourtant pas un questionnement nouveau. Elle s'inscrit dans la suite de plusieurs tentatives antérieures qu'avait suscitées depuis plus d'une trentaine d'années la lecture de certains textes d'Aristote chez les maîtres de Paris et d'Oxford. Toute l'argumentation de Thomas sera pour montrer que s'il a à penser Dieu, l'être humain le pense humainement, c'est-à-dire, comme le note Chenu, "selon le procédé discursif qui est le sien" 5 . Et la théorie de la subalternation que propose alors Thomas aura pour conséquence de bien marquer cl'intériorité effective du travail rationnel du théologien dans le donné révélé" 6 . Michel Corbin résume très bien le projet thomasien déjà dans son commentaire de Boèce lorsqu'il écrit: "ll s'agissait, pour ainsi dire, de faire entrer en doctrine de la foi les exigences totales de la raison, et non de justifier, face à la philosophie comme oeuvre de la raison, l'existence de cette doctrine" 7 . Le projet trouvera comme son achèvement dans la Somme théologique, mais déjà dans ce commentaire de Boèce doit-il le défendre contre ceux qui l'accusent rien de moins que de couper d'eau le vin fort de la parole et de la sagesse divines.

L'accusation est extrêmement sérieuse. Elle laisse entendre que le projet thomasien de l'inscription de la raison dans la foi ne conduit à rien d'autre qu'à une dissolution de cette dernière qui, pour demeurer ce

historique), $2 / 1962$ (1944), p. 507 . Il semble bien que de tout temps l'inscription scolaire de la pensée chrétienne ait indisposé des croyants. Déjà au III ${ }^{e}$ siècle, Clément d'Alexandrie se plaignait de «certains ignorants qui s'effraient du moindre bruit, à savoir que l'on doit s'en tenir aux choses essentielles, à celles qui ont du rapport à la foi et que l'on doit négliger celles qui viennent du dehors» (Strom. I, I, 18.2), et de ceux «qui se croient de l'esprit et qui estiment qu'on ne doit se mêler ni de philosophie ni de dialectique, ni même s'appliquer à l'étude de l'univers; ils réclament la foi pure et simple, comme s'ils refusaient de travailler la vigne et voulaient immédiatement cueillir les raisins». (Strom. I, IX, 43.1) [Trad. J. Lebreton, dans Histoire de l'Église. T. 2. Sous la direction de A. Fliche et V. Martin. (s.l.), Bloud et Gay, 1938, p. 238.]

5 M.D. Chenu, La théologie comme science au XIIIe siècle, p. 69.

6 Ibid., p. 83.

7 M. Corbin, Le chemin de la théologie chez Thomas d'Aquin. Paris, Beauchesne, (Bibliothèque des Archives de philosophie, NS \#16) , 1974, p. 438. 
qu'elle est, ne pourrait pas s'affirmer comme travail de la raison dans toute son épaisseur historique. A la limite, "foi" et "raison" nommeraient deux régimes différents, hiérarchiquement superposés, où la "raison» ne serait tout au plus qu'«au service» d'une foi, déjà toute constituée et lui commandant de l'extérieur. Pour précise qu'elle soit, cette objection, qui refera constamment surface, et souvent dans les mêmes termes, dans l'histoire de la théologie, ne trouve pas ses assises dans ce qu'on appelle parfois la grande tradition de la pensée chrétienne ${ }^{8}$. Elle ne peut s'appuyer ni sur Augustin, ni sur Anselme de Cantorbéry si l'on ne veut nommer que deux moments importants avant Thomas d'Aquin' ${ }^{9}$. Peut-être se veut-elle interprétation de l'idée paulinienne de la "folie du monde" face à la "sagesse de Dieu»10. Elle accompagnera en tout cas jusqu'en ce $X X e$ siècle finissant tout projet

8 On la retrouve tout récemment dans un article du juriste catholique M. Kriele, «Aktuelle Probleme des Verhältnisses von Kirche und Staat», Internationale katholische Zeitschrift 19 (Nov. 1990):541-555: pour Kriele, puisque de plus en plus de théologiens universitaires n'ont plus la foi, on doit procéder peu à peu au démantèlement des facultés de théologie en retirant progressivement la permanence aux professeurs au fur et à mesure du renouvellement du corps professoral, refusant aux récalcitrants la compétence pour former les futurs prêtres et les agents de pastorale et les laissant «discuter sans fin du doute et changer le vin en eau» (p. 553). Une des grandes inquiétudes de Kriele consiste très précisément dans l'introduction des sciences humaines en théologie: «À la place de la foi s'installent la psychologie, l'anthropologie, la sociologie, la mythologie, les sciences historiques ou l'engagement humanitaire et politique.» Ce refus de la rationalité modeme et la crainte de voir la foi s'y dissoudre demeure quelque chose de latent dans plusieurs cercles catholiques. On en retrouve un autre exemple tout aussi inquiétant dans A.M. Sicari, «La sainteté de l'auteur et l'orthodoxie du discours», Revue catholique internationale Communio 6 (sept.-oct. 1981): 20-28. Ici aussi les sciences humaines en théologie demeurent explicitement une pierre d'achoppement: «on doit à un moment donné accepter de les "briser", de les "crucifier" ou même de s'en dissocier» (p. 26). Évocation des objections contre la "scientificité» de la théologie aussi dans A.I.C. Heron, «La théologie comme science?», Revue Thomiste 85 (1985): 231-232. Aussi l'avant-propos de J.-Y. Lacoste, pp. 229-231.

9 Pour Anselme voir l'étude importante de K. Kienzler, Glauben und Denken bei Anselm von Canterbury. Freiburg-Basel-Wien , Herder, 1981, 413 p.

10 Cette polarité paulinienne (1 Co. 1) a souvent servi à mettre en garde contre ce qu'on estimait être des prétentions inacceptables de la théologie. Cette argumentation affleure, p. ex., dans un texte de Rosmini que cite Sicari pour introduire sa problématique ( lec. cit., p. 21) et Sicari la reprend expressément à son compte (p. 23). On aurait encore intérêt à relire sur cette question les analyses de $\mathrm{H}$. Schlier, «Kerygma et Sophia. Contribution au fondement néo-testamentaire du dogme», dans Le temps de l'Église. Recherches d'exégèse. Tournai, Casterman, (Cahiers de l'actualité religieuse \#14), 1961, 212-237. 
d'articulation en régime chrétien de la raison et de la foi, et si elle a pu servir à exprimer en certaines périodes l'idée que l'on se faisait de la radicalité et de l'originalité de la foi chrétienne, on y reconnaîtra hélas! le plus souvent l'écho d'une foi angoissée à l'idée d'avoir à s'inscrire dans le même terreau que Celui qu'elle confesse, en pleine épaisseur humaine. C'est qu'on aura oublié la réponse d'Augustin à son correspondant Consentius: "A Dieu ne plaise que la foi nous empêche de recevoir ou de demander la raison de ce que nous croyons! Nous ne pourrions pas croire si nous n'avions pas des âmes raisonnables»11. Celle de Thomas n'est pas de moins grande portée: dans l'usage que les théologiens font de la philosophie, s'ils savent bien travailler, ce n'est pas le vin qui est coupé d'eau, mais bien l'eau qui est changée en vin.

\section{Une exigence de la foi}

En reprenant à son compte cette image biblique, Thomas ne réglera évidemment pas la question pour les générations à venir. Il contribuera peut-être même au contraire à nourrir la suspicion d'autres générations de philosophes qui ne voudront rien savoir d'une théologie qui semble prétendre ainsi les utiliser selon ses goûts et ses besoins. II indique cependant très vigoureusement à quel niveau se nouent les rapports entre la foi et la rationalité humaine. Ce que Thomas veut montrer, c'est que la raison humaine appartient à la foi, qu'elle accède même chez elle à sa vérité, qu'elle lui est intérieure, mais elle l'est comme raison humaine, avec tout ce que cela signifie. En d'autres termes, le projet thomasien ne consiste pas à asservir la raison où à soustraire, en la transfigurant, la foi à ses exigences mais, pour le redire encore une fois avec les mots du P. Chenu, à assurer al'intériorité effective du travail rationnel du théologien dans le donné révélé»12.

Cet élément du projet thomasien de théologie me paraît fondamental pour la question qui nous occupe. Dans la conception que Thomas propose de la théologie, on s'est habitué à retenir avant tout son armature aristotélicienne. C'est en effet en dépendance du concept de science transmis par les écrits d'Aristote que Thomas en vient à interpréter la théologie comme une science. Moins attentif à la radicale transformation qu'il fait alors subir à ce concept, de manière à ce qu'il

11 Lettre CXX. (Lettres de Saint-Augustin. Traduites en français et précédées d'une introduction par Poujoulat. T. II.. Paris, J. Lefort, 1858, p. 329)

12 La théologie comme science au XIII siècle, p. 83. 
puisse traduire une authentique exigence ef une réelle possibilité de la foi elle-même, on a surtout retenu le type de rationalité qui a continué malgré tout de se maintenir à l'oeuvre en théologie et la pratique scientifique qu'elle génère habituellement. Mais depuis le XIlle siècle de Thomas d'Aquin, non seulement l'idée aristotélicienne de science mais tout le régime de la rationalité qui l'a nourrie ont été profondément modifiés. La notion aristotélicienne de science n'est plus apte à rendre compte de la pratique scientifique contemporaine. La critique de la pensée métaphysicienne a creusé un fossé, à certains égards infranchissable, entre la "raison" médiévale et la "raison" moderne. $\mathrm{Ce}$ qui pouvait permettre à Thomas d'Aquin d'interpréter la théologie comme une science selon les catégories aristotéliciennes ne nous est plus réellement disponible comme une possibilité authentique pour comprendre notre propre expérience ${ }^{13}$.

Mais ce qui valait pour Thomas et qui redevient clair à notre époque c'est que la foi chrétienne ne délimite pas un espace déjà tout constitué à côté ou au-dessus de celui de la rationalité quotidienne, que le croyant habiterait aussi par ailleurs et dont il mettrait éventuellement les ressources à profit pour faire à son gré le trajet de l'un à l'autre. La théologie ne doit pas être comprise comme l'intermédiaire scientifique qui permettrait de relier ces deux domaines, selon les intérêts du moment. Toute une interprétation du prétendu conflit entre la foi et la raison qui traverse, depuis la Contre-Réforme, l'histoire de la théologie et même plus largement l'histoire de la pensée occidentale, se nourrit de cette caricature. Le défi auquel la théologie se trouve toujours confrontée est, tout à l'inverse, de montrer que la foi chrétienne, loin d'ouvrir un espace paralièle ou supérieur, est une manière d'habiter le seul monde de l'homme et qu'elle se trouve ainsi traversée de part en part, non pas comme d'un handicap provisoirement inévitable, mais comme d'une exigence de nature, par une raison humaine en travail d'interprétation. C'est ce qui est à la source du projet thomasien de théologie, bien avant ses emprunts à la philosophie de son temps: cette

13 Littérature désormais incontrôlable sur cette question. À titre d'exemple, du point de vue de la pratique scientifique contemporaine: T.S. Kuhn, La structure des révolutions scientifiques. Paris, Flammarion, 1972 (1962), 246 p. Du point de vue de la théologie: B. Welte, "Uber den Sinn, die Notwendigkeit und die Grenzen einer "Enthellenisierung" des Christentum», dans Zwischen Zeit und Ewigkeit. FreiburgBasel-Wien, Herder, 1982, 233-248. J.S. O'Leary, Questionning Back. The Overcoming of Metaphysics in Christian Tradition. Minneapolis-Chicago-New York, Winston Press, 1985, 225 p. 
conviction que la foi chrétienne est un audacieux projet d'existence dans le monde, capable de se proposer en raison et de se mesurer avec vigueur à d'autres projets.

\section{Une théologie en dialogue avec la pensée de son temps}

Ce qui est tout à fait remarquable chez Thomas d'Aquin, et on pourrait, je crois, en dire autant d'Origène et d'Augustin, c'est que cette conviction va trouver à s'expliciter à même le dialogue qu'il instaure avec la pensée de son temps ${ }^{14}$. Celle-ci deviendra en quelque sorte le lieu de cette reprise créatrice de l'exigence permanente de la foi d'inscrire son pari interprétatif au coeur même de l'expérience que les contemporains font d'eux-mêmes et de leur monde. Et pourtant, ceci ne devrait pas étonner car il n'y a pas d'autres lieux où la foi trouverait à se déployer. Ce n'est qu'après le XIlle siècle, et à la mesure même de sa dérive par rapport aux questions et aux ressources vives de son temps, que la théologie se déploie en enseignement répétitif d'une doctrine et se fige en systèmes. Croyant pouvoir s'affranchir des interrogations de son temps, elle éprouve alors bien plus le besoin de se protéger contre une pensée qui lui paraît de plus en plus étrangère et ainsi menaçante, laissant la foi s'affirmer en "zone d'accès contrôlé", que celui de réouvrir chaque présent à ses plus proches possibilités, dont la question de Dieu demeure le chemin le plus mystérieux mais sans doute aussi le plus permanent.

Ce n'est pas en cherchant à se réaffirmer comme "science", au sens de la rationalité scientifique moderne, que la théologie pourrait légitimer sa place au sein'de l'université, si du moins l'université ne se trouve pas définie par cette seule rationalité. Un projet de scientificité pourrait trouver à s'affirmer d'une manière purement formelle et comme en extériorité par rapport à la véritable prétention de la théologie et, partant, de la foi. Certains traités de soi-disant théologie dogmatique ont très bien réalisé au XVIIle et XIXe siècles l'exigence scientifique de leur époque, mais pour finalement creuser l'écart entre les appels de

14 Pour Thomas: «La Somme Théologique de Thomas d'Aquin est probablement le plus significatif et le plus grandiose exemple de ce mode de rencontre entre la foi chrétienne et la culture philosophique profane, parce que la rencontre y est comprise comme essentielle à la théologie ... " (M. Corbin, "Le chemin de l'interprétation", dans L'Inoui de Dieu. Six études christologiques. (s.1.), DDB, 1980, p. 25. 
l'expérience humaine dans le monde et les possibilités qu'offrait l'interprétation croyante. C'est fondamentalement au titre de sa capacité à proposer une interprétation féconde et inédite de l'existence possible dans le monde qui soit d'elle-même en état de se mesurer à d'autres interprétations que la théologie peut aujourd'hui trouver place à l'université.

Cela ne signifie pas que la théologie n'a pas à proposer son interprétation selon des critères de logique ou de rigueur reconnus comme tels par les contemporains. Cela non seulement doit-elle le faire si elle veut prétendre à quelque crédibilité mais elle est tout à fait capable de le faire, même s'il s'agit d'une tâche qui demeure sans cesse à recommencer. À cet égard, la théologie contemporaine est beaucoup plus consciente des implications méthodologiques et herméneutiques de son discours que ne l'a été celle des siècles précédents. Et la quantité désormais incontrôlable de travaux de toutes sortes sur ces questions en théologie, si elle témoigne d'une crise certaine, que la théologie partage largement d'ailleurs avec la philosophie et les sciences humaines ${ }^{15}$, témoigne également de sa capacité à s'inscrire dans le dialogue scientifique contemporain. Mais là n'est pas tout à fait la question. Il ne s'agit pas d'abord pour la théologie d'affirmer son caractère "scientifique" mais de faire en sorte que le travail de compréhension et de reprise rationnelle apparaisse comme une exigence et une possibilité internes à la foi, c'est-à-dire sans lesquelles la foi n'est plus foi.

C'est pour avoir entretenu fallacieusement une coupure entre foi et "raison" que la théologie s'est pendant de longs siècles retrouvée en porte-à-faux par rapport à la pensée de son temps. C'est aussi ce qui a largement contribué aux exclusions dont elle a été victime au temps de l'Aufklärung et dont le projet fitchtéen d'université est une belle illustration ${ }^{16}$. II faudra bien qu'un jour on se mette sérieusement à la

15 Cf. p. ex.: R. Sainsaulieu, «Crises de société, défi pour les sociologues», dans Éclatement des savoirs et nouvelles cohérences. Paris, Centre Sèvres, (Travaux et Conférences du Centre Sèvres \# 8), 1986, 7-18. P. Germain, «Sur quelques caractéristiques des disciplines scientifiques et sur la portée de la science», Les Études philosophiques (2/1978): 157-170. J. Ladrière, "Les sciences humaines et le problème de la scientificité», Ibid., pp. 131-150.

16 Voir le projet d'université que Fichte avait conçu pour une nouvelle université qui devait être érigée à Berlin, dans: J.G. Fichte, Sämmtliche Werke. Band 8. Berlin , 
tâche pour dégager les intérêts qui ont joué dans ce long et complexe processus de dérive de la théologie par rapport à la pensée de son temps et essayer de comprendre tout le poids qui a pesé sur la théologie du fait de la reprise en philosophie au XVIIle siècle des grandes questions théologiques traditionnelles. Peut-être comprendra-t-on mieux alors pourquoi et de quelle manière la théologie est devenue progressivement une discipline explicitement et directement au service d'une institution ecclésiale et comment s'est produite l'occultation qui s'en est suivie de son rapport premier à la foi. II ne s'agit évidemment pas de nier que la théologie ait comme telle un rapport essentiel à l'Église mais de bien voir qu'elle est avant tout une exigence de la foi et que c'est ainsi qu'elle doit s'offrir à l'attention des contemporains ${ }^{17}$.

\section{Une théologie de part en part historique}

Exigence de la foi, c'est à même les ressources et les exigences du moment que la théologie traduit son inscription historique et manifeste qu'elle a sans cesse à se reprendre pour chaque présent. II faut ici réaffirmer avec force que le théologien ne peut plus aujourd'hui se prévaloir d'un statut épistémologique qui serait propre à sa science théologique. Comme le rappelle fort à propos Jean-Paul Gabus:

“Dans la mesure où l'intelligence théologique se
découvre elle-même historiquement et contextuellement
située et qu'elle est reçue comme telle par la culture
ambiante, cette intelligence ne peut que renencer à tout
dogmatisme. En particulier, elle se doit de renoncer à
formuler un discours qui prétendrait être le reflet
homogène ou l'écho fidèle d'une Révélation qui
échapperait comme ce discours à l'historicité radicale du
savoir humain. II n'est épistémologiquement plus

Verlag von Weit und Comp., 1846, 97-204. La théologie était explicitement exclue de cette nouvelle université.

17 Ceci a plusieurs conséquences concrètes. Le fait, par exemple, que pendant très longtemps la théologie n'ait été que l'affaire des clercs et qu'encore aujourd'hui on l'envisage en plusieurs milieux comme étant essentiellement au service de la formation des futurs prêtres et des agents de pastorale montre qu'on a perdu de vue la source de l'exigence théologique. Le texte de Kriele cité plus haut n. 8 est un bel exemple de ce rétrécissement de la fonction théologique. On comprend qu'il soit difficile de prendre alors bonne mesure et du rapport de la théologie avec la pensée de son temps et des modifications énormes qui se sont opérées presque partout en Occident depuis une trentaine d'années dans la clientèle des Facultés de théologie. 
possible aujourd'hui de fonder le discours théologique sur l'hypothèse d'une prétendue homogénéité entre le donné révélé et le dogme (thèse de A. Gardeil et du néothomisme) ou encore d'une éventuelle participation du théologien à la connaissance que Dieu a de lui-même par un acte libre et souverain de sa grâce (thèse de Karl Barth et de son école)" 18 .

Il y a quelque chose qui ressemble à une trace de dinosaure dans un paysage du $X X_{\theta}$ siècle dans l'affirmation qui veut que "la théologie parle de Dieu et avec Dieu comme les anges dans le Ciel»19! Cette conscience très vive de l'inscription historique du discours théologique, dont il serait aisé de multiplier les témoignages dans la théologie du XXe siècle ${ }^{20}$, n'est pas le résultat d'une concession stratégique à l'esprit du temps. Elle repose ultimement sur une compréhension de la foi ellemême dont elle redécouvre, par-delà quelques siècles qui l'avaient plutôt oubliée, le sens qu'elle avait dans la Bible et qu'elle a continué d'avoir dans la tradition patristique et dont se souvenait encore - beaucoup plus qu'on ne le pense généralement - Thomas d'Aquin.

II est assez évident que cette prise de conscience pose un certain nombre de questions, à la vérité fondamentales, que je ne peux pas traiter ici. Mais il importait d'y insister car elle indique à mon sens ce qu'on pourrait peut-être appeler la "condition de possibilité" de l'inscription universitaire de la théologie en même temps qu'elle marque l'enjeu auquel elle s'y trouve confrontée.

Inscrite en histoire et devant articuler son discours à même les possibilités de son époque, la théologie se trouve de ce fait affectée par le pluralisme qui caractérise la situation actuelle de la pensée et les

18 J.P. Gabus, Critique du discours théologique. Neuchâtel-Paris, Delachaux et Niestlé, (Bibliothèque théologique), 1977, p. 22. Voir aussi: P.-L. Dubied, «La place d'une Faculté de théologie dans l'université aujourd'hui», Revue de théologie et de philosophie 120 (1988):21-28 (24).

19 R.L. Bruckberger, «Dialogue théologique», dans: Dialogue théologique. Pièces du débat entre «La Revue Thomiste» d'une part et les R.R. P.P. de Lubac, Daniélou, Bouillard, Fessard, von Balthasar, S.J., d'autre part. Saint-Maximien, Les Arcades, 1947, p. 9.

20 Pour la théologie d'expression française cf. J.C. Petit, «La compréhension de la théologie dans la théologie française au XXe siècle», Laval théologique et philosophique 45 (1989): 379-391; 47 (1991): 215-229; 48 (1992): 415-431. 
déplacements de la raison qui s'y sont opérés. Non seulement la théologie ne trouve plus devant elle un interlocuteur unique qui incarnerait la philosophie de son temps, mais elle ne se trouve plus liée par une seule possibilité philosophique pour penser son interprétation de l'humain dans son monde ${ }^{21}$. Les déplacements qui affectent l'expérience croyante elle-même l'empêchent de se concentrer sur une seule tradition philosophique. Sa pertinence ne se décide pas d'avance, à partir de l'autorité d'une seule tradition ou d'une institution qui jouirait encore d'une quelconque autorité. Sa fonction ne consiste pas non plus à affirmer la foi ou à y préparer - c'est là le rôle conjugué de la liturgie et de la catéchèse - ni à proprement parler à la défendre, car elle ne s'estime pas à priori en situation de combat. Sans cesse renvoyée à une pratique croyante, la théologie se situe au niveau d'un discours second, discours de la foi certes, mais discours de reprise et d'interprétation critique qui entend manifester à même la rationalité de son temps la pertinence et la fécondité actuelles de l'interprétation croyante pour la compréhension que l'homme contemporain peut avoir de lui-même et de son monde.

\section{Une possibilité actuelle}

Certes, dans toute une dimension de son travail, la théologie contribue largement à "maintenir en éveil une part décisive de notre conscience collective"22. Cette tâche risque d'apparaître de plus en plus nécessaire au fur et à mesure que s'affirme le caractère amnésique qui marque la civilisation technicienne dont l'envahissement devient chaque jour plus évident. Mais là n'est pas la tâche première de la

21 Sur la question du rapport entre la théologie et le pluralisme contemporain, parmi une littérature très abondante: C. Geffré, «Pluralité des théologies et unité de la foi», dans Initiation à la pratique de la théologie. T. I: Introduction. Paris, Cerf, 1982, 117 142 (Bibliographie). Pour une approche sociologique du pluralisme: H. Gross, «Zum Begriff der pluralistischen Gesellschaft», dans: Zeugnisse. Theodor W. Adorno zum sechzigsten Geburtstag. (HORKHEIMER, M., hrsg.), Frankfurt-am-Main, Europäische Verlagsanstalt, 1963, 439-450. Les travaux de Jean Ladrière peuvent servir d'illustration d'un travail théologique réalisé dans ce nouveau contexte: p. ex. J. Ladrière, L'articulation du sens. T. 1, Discours scientifique et parole de la foi. T. 2, Les langages de la foi. Paris, Cerf, (Cogitatio Fidei \# 124 et 125), 1984 . Cf. J.-F. Malherbe, Le langage théologique à l'âge de la science. Lecture de Jean Ladrière. Paris, Cerf, (Cogitatio Fidei \# 129), 1985.

22 P.-L. Dubied, loc. cit. (n. 16), p. 25. Voir aussi R. Puza, «Staatskirchenrechtliche und kirchenrechtliche Stellung und Aufgaben der Theologischen Fakultäten», Theologische Quartalschrift 171 (1991):108-114 (112). 
théologie qui ne peut pas être réduite à une simple science historique chargée d'étudier des traditions jadis importantes dans l'histoire de l'Occident, dont la compréhension pourrait à certains égards nous être nécessaire pour comprendre notre propre présent mais qui s'éloigneraient aussi de plus en plus de nous au rythme des transformations de notre présent.

Sa tâche primordiale est d'expliciter et d'éprouver une possibilité actuelle d'existence dans le monde et de le faire en ouvrant un espace de libre communication entre ceux qui pensent, de manière à ce que la proposition que la théologie estime pouvoir faire s'offre à partir d'ellemême et non pas à partir des intérêts qu'un groupe ou une institution pourrait $y$ trouver.

La proposition que fait la théologie se noue dans ce que nous nommons la "question de Dieu" et dans la forme que prend cette question dans la figure de Jésus de Nazareth. Inscrite en université, la théologie prétend que cette question demeure en travail au coeur de l'existence humaine dans le monde; qu'elle peut être non pas épuisée mais articulée en discours sensé, accessible à la réflexion commune; qu'elle a même une fonction intégratrice, peut-être encore plus aujourd'hui, alors que les pièces du casse-tête humain se multiplient mais semblent de moins en moins s'emboîter les unes dans les autres. La théologie prétend surtout que cette "question de Dieu", jusque dans sa figure en l'homme Jésus, est une authentique question, ouverte sur un véritable espace de liberté. C'est un fruit pervers de l'impérialisme de l'esprit grec sur la tradition chrétienne que d'avoir petit à petit refermé sur une intemporelle certitude la quête croyante ouverte dans l'autre grande tradition de l'Occident qu'est le judaïsme ${ }^{23}$. La figure de la foi chrétienne ne doit pas être cherchée dans le théologien "qui parle avec Dieu comme les anges dans le Ciel" mais aujourd'hui comme hier dans la figure d'Abraham qui, "répondant à un appel", se mit en chemin, "sans savoir où il allait" et "venant habiter à l'étranger" (Héb. 11,8-9).

C'est le grand défi de la théologie. Au moment où elle met en oeuvre toute la rigueur qu'exige son objet et qu'attendent les contemporains: le défi de ne pas transformer en gnose l'audacieuse interprétation que la foi

23 Cf. B. Welte, «Die Lehrformel von Nikaia und die abendländische Metaphysik», dans Zur Frühgeschichte der Christologie. Ihre biblischen Anfänge und die Lehrformel von Nikaia. (WELTE, B., hrsg.). Freiburg-Basel-Wien, Herder, (Qaestiones Disputatae \# 51), 1970, 100-117. 
fait de l'existence humaine et de la proposer de telle manière qu'elle apparaisse aujourd'hui comme une authentique possibilité de se comprendre soi-même autrement. II s'agit là d'un véritable défi car, pour les contemporains, la théologie est encore trop souvent la face savante d'une gnose ancienne qui saurait d'avance et sans discussion ce qui nous, les humains, nous concernerait au premier chef. La théologie de la foi chrétienne demeure, certes, de part en part redevable d'une expérience particulière et de sa tradition plurielle. Je reviens là-dessus à l'instant. Mais il importe de réaffirmer, simplement mais clairement, qu'à l'instar de tous ses collègues qui cherchent comme lui quelque réponse aux interrogations que pose notre existence d'homme et de femme dans le monde, le théologien, pour le dire avec Pierre Gisel,

“ne part pas d'un point situé hors de l'histoire, postulant un type de rapport direct avec Dieu (conquis ou reçu), vivant d'un lien immédiat à la vérité. La foi et la théologie déchiffrent la vérité au creux des vagues de l'histoire. Mais elles le font en se référant à une tradition spécifique. La foi et la théologie chrétiennes lisent et interprètent le combat et l'espérance déterminée qui se manifestent dans telle chaîne de figures historiques. Comme ces figures, elles demeurent elles-mêmes historiques, inachevées, dans l'ignorance des premiers commencements et des fins dernières. Elles ne sont pas liées à un savoir secret des causes, mais à un mode d'exister, d'habiter et de structurer le monde. Certes, elles parient pour la valeur "exemplaire" de cette chaîne historique. Mais elles ignorent toute synthèse universelle, toute identité commune au-delà des différences. Si elles peuvent proposer une "réponse" à l'attente et à l'aspiration du monde, c'est uniquement en fonction d'une Promesse qui tout à la fois donne sens à ces figures et que manifestent ces figures. Rien de plus. Mais rien de moins" 24 .

Il devrait être clair aux contemporains, à même sa manière de travailler, que la théologie se reconnaît dans les paroles d'Augustin qui avoue franchement ne pouvoir dire

24 P. Gisel, Vérité et Histoire. La théologie dans la modernité. Ernst Käsemann. Paris, Beauchesne, (Théologie historique \# 41) ,1977, p. 347. 
“lui aussi [que] ce qu'il peut, car qui est capable de dire ce qui est? J'ose l'affirmer, mes frères, Jean [l'évangéliste] lui-même peut-être n'a pas dit ce qui est, mais il a dit lui aussi ce qu'il a pu, car il n'était qu'un homme et il a parlé de Dieu, un homme inspiré de Dieu sans doute, mais néanmoins un homme. Parce qu'inspiré, il a dit quelque chose; sans l'inspiration, il n'aurait rien dit; cependant, parce que l'inspiré était un homme, il n'a pas dit tout ce qui est, mais il a dit ce qui était possible à un homme» 25 .

Augustin pensait sans doute à cette limite qu'impose à notre connaissance notre nature d'être fini 26 . On ne peut pas dire que la théologie a toujours fait preuve de la même lucidité ou tout au moins de la même franchise. Quant à nous, nous avons quelques raisons supplémentaires pour ne pas défendre l'intérêt de la théologie en la fondant dans une connaissance éternelle à laquelle une mystérieuse révélation lui aurait assuré un accès des plus sûrs. Peut-être mieux que ceux qui l'ont précédé, l'homme moderne a appris que tout n'est pas possible de la même manière, toujours et partout. Une théologie qui procéderait comme si elle n'était pas traversée de part en part par cette expérience entretiendrait de bien tristes illusions.

\section{Du savoir à la recherche}

Des transformations profondes dans l'expérience que l'être humain fait de lui-même et de son monde ont opéré des déplacements majeurs dans les ressources de l'interprétation. L'émergence d'une nouvelle conscience historique, l'expérience douloureuse des limites d'une raison instrumentale, la brisure violente d'un monde cassé entre les riches et les pauvres et la montée croissante d'une menace qui paraît de plus en plus concerner les possibilités mêmes de la vie sur notre planète et qui rend en tout cas chaque jour davantage aléatoire son avenir comme monde humain, voilà autant de réalités qui ont progressivement orienté le travail de la théologie dans des directions bien différentes de celles qui lui étaient devenues familières au cours des derniers siècles.

25 Tractatus in Johannis Evangelium. I, 1. [ (s.l.), Desclée de Brouwer, (Oeuvres de saint Augustin, 71), 1969, p. 129]

26 Cf. aussi Thomas d'Aquin: «La révélation ne nous élève pas à un autre mode de connaître que celui qui tire son origine du sensible.» (In Boet. de Trin. q. 6, a.3) 
Ceci est particulièrement visible dans l'émergence de lieux nouveaux où s'articule le travail de la théologie. Ici on peut certainement nommer le nouveau rapport qui est en train de s'établir en théologie avec les sciences humaines ${ }^{27}$. On a déjà évoqué plus haut le pluralisme qui définit désormais le monde de la théologie. Mais le déplacement dans lequel se trouve irréversiblement engagée la théologie est peut-être surtout visible à l'attention renouvelée à ce qui a, dès l'origine, constitué le lieu théologique par excellence ${ }^{28}$ et qui était depuis longtemps disparu à l'arrière-plan, à savoir la pratique chrétienne ${ }^{29}$. C'est en attirant l'attention sur cette question que je voudrais conclure mon propos car elle me paraît fort bien illustrer le défi qui se pose aujourd'hui en théologie, parce que c'est d'elle que la théologie reçoit aujourd'hui sa tâche d'“intellectus fidei» et qui inscrit celle-ci dans le registre de la "quaestio" plutôt que de la "scientia".

La théologie estime, en effet, devoir sérieusement prendre en compte la pratique croyante. Elle le fait parce qu'il est devenu clair que loin d'être un champ d'application d'une théorie acquise et structurée par ailleurs, la pratique croyante possède, comme le dit Geffré, "un rôle structurant dans l'élaboration du message»30. Plus largement encore, c'est la pratique humaine comme telle qu'elle entend prendre en compte, car la pratique humaine est elle-même le lieu de production d'un savoir auquel l'être humain ne peut nulle part ailleurs accéder. C'est d'ailleurs de cette expérience dont témoigne la tradition biblique. II n'y a pas pour elle d'autre lieu pour accéder à la foi qu'une pratique particulière. À notre époque les diverses théologies de la libération ne reposent pas sur une autre conviction.

27 Cf. par. exemple. J.-B. Metz, «Die Verantwortung der Theologie in der gegenwärtigen Krise der Geisteswissenschaften», dans Wissen als Verantwortung. Ethische Konsequenzen des Erkennens. (MULLER, H.P., hrsg.), Stuttgart-BerlinKöln , W. Kohlhammer, 1991, 113-126.

28 C'est ce qui se trouve affirmé par exemple dans la réinterprétation johannique de la 'emeth ("aletheia"/"vérité") vétéro-testamentaire.

29 Parmi une littérature déjà abondante: Le déplacement de la théologie. Paris, Beauchesne, (Le point thénlogique \#21), 1977, 184 p. Pratique et théologie. (GISEL, P., éd.), Genève, Labor et Fides, (Pratiques \# 1), 1989, 224 p. E. Schillebeeckx, L'histoire des hommes, récit de Dieu. Paris, Cerf, (Cogitatio Fidei \#166), 1992 (1989), $381 \mathrm{p}$.

30 C. Geffré, «Du savoir à l'interprétation», dans Le déplacement de la théologie, p. 62 . 
Lieu de la foi et déjà interprétation de la foi, la pratique croyante n'épuise cependant pas la foi et elle n'en est pas la dernière définition. C'est ici, en définitive, que tout risque de se jouer quant à la compréhension que nous pouvons avoir de la théologie. La foi chrétienne ne s'épuise pas dans une praxis ou une manière de vivre ou de comprendre notre existence humaine. On ne pourrait en tout cas le prétendre tout en se réclamant de l'expérience dont témoigne la tradition biblique et de l'interprétation qu'en a donnée la tradition théologique. Si l'on veut prendre le risque d'une brève formule, on peut dire que la foi chrétienne c'est la re-connaissance qu'en Jésus le Christ se manifeste la vraie figure de Dieu, que dans la vie, la mort et la résurrection d'un homme se manifeste le mystère indicible qu'on appelle Dieu. Revendiquée par cette médiation, la théologie "n'a point à se soucier de devenir système et à rêver de nouvelles sommes scientifiques" 31 . Elle se voit cependant confiée la tâche de parler de ce Dieu, de le faire de telle manière qu'apparaisse ce que cela signifie qu'il se révèle dans une figure humaine. Le défi de la théologie, c'est de le faire de telle manière que devienne visible ce qu'il en va de notre existence d'homme et de femme dans cette révélation humaine de Dieu. Si la théologie y arrive, elle réalisera ce qu'aucune autre discipline ne peut réaliser, mais elle pourra ainsi rencontrer ce qui se cherche présentement de meilleur dans les autres disciplines ${ }^{32}$, qui se trouvent aussi concernées par la question que l'être humain ne cesse d'être à lui-même et par ce qui s'y montre lorsqu'il s'y engage. Ce qui se trouve alors confié à la théologie ne devrait plus être pensé comme un grand vin qu'elle doit bien se garder de couper mais comme une question, c'est-à-dire à proprement parler comme une quête, qu'elle devra éviter d'étouffer ${ }^{33}$. Aujourd'hui peutêtre plus qu'hier, la théologie en université ne peut se permettre d'oublier que "chaque question possède une force que la réponse ne contient plus" et que finalement ce qu'il faut demander à Dieu, ce n'est pas tellement les bonnes réponses que "de nous donner la force de pouvoir lui poser de vraies questions" 34 . Mais ne serait-ce pas là, finalement, le vrai sens de la "recherche" et de l"censeignement" qu'elle suscite?

31 M. Corbin, "Garder la parole de Dieu. Essai sur Luc 11,28», dans Le déplacement de la théologie, p. 117.

32 Cf. Corbin, ibid., p. 118.

33 Cf. J.C. Petit, «Théologie et expérience», dans L'expérience comme lieu théologique. ( LACELLE , E et Th. POTVIN., éd.), Montréal , Fides, (Héritage et Projet \#26), 1983, 13-30.

34 Elie Wiesel, La nuit. Paris, Éd. de Minuit, 1958, pp. 17-18. 


\section{Théologie et université 35}

XXX. 1988. "Statement of Presidents of Leading Catholic Universities of North America on the Scheme for a Proposed Document on the Catholic University", dans Beadings in Moral Theology. No. 6: Dissent in the Church. (CURRAN, C.E. and R.A. McCORMICK, éd.), New York-Mahwah, Paulist Press, 271-275.

AZEVEDO, M. DE C.1990. "L'université catholique et la transmission de la foi chrétienne", dans Défis présents et à venir de l'université catholique. Actes du colloque international du centenaire de I'Université Saint-Paul, 28-31 août 1989. Ottawa, Université Saint-Paul, 167-186.

BONE, E. 1988. "L'Université catholique. Une difficile vocation", Études $368: 531-541$.

BURGE, E.J. 1982. "Higher Degrees in Theology, 1950-1974. A Statistical Analysis and a Commentary", Theology 85: 114-120.

COSMAO, V. 1990. "L'université catholique et la pratique de la foi chrétienne", dans Défis présents et à venir de l'université cathelique. Actes du colloque international du centenaire de I'Université Saint-Paul, 28-31 août 1989. Ottawa, Université Saint-Paul, 209-219.

DAIBER, K.-F. 1990. “Der institutionnelle Rahmen als Determinante von Universitätstheologie und kirchlicher Praxis", Pastoraltheologie 79: 302-317.

DEUMLAND, K.D. 1987. "Les grades académiques et l'Église en Allemagne fédérale", Praxis juridique et religion 4: 69-76.

DEZZA, P. 1979. "La Constitution Apostolique "Sapientia christiana", Nouvelle Revue Théologique 101: 740-752.

DUBIED, P.-L. 1988. "La place d'une Faculté de Théologie dans l'Université aujourd'hui', Bevue de Théologie et de Philosophie 120: 21-28.

DULLES, A. 1989. "University Theology as a Service to the Church", Thought 64: 103-115.

35 Mise à jour de: J.-C. Petit, La théologie. Sa nature, ses méthodes, son histoire, ses problèmes. Répertoire bibliographique international. Montréal, Les Presses de I'Université de Montréal, 1979, 407-421. 
EBELING, G. 1975. "Zur Existenz theologischer Fakultäten an staatlichen Universitäten", dans EBELING, G. Wort und Glaube. III, Beiträge zur Fundamentaltheologie, Soteriologie und Ekklesiologie. Tübingen, J.C.B. Mohr (Paul Siebeck), 164-169.

EPP, R. 1990. "Aperçu sur les Facultés et les écoles de théologie catholique en France au XIXe siècle", Revue des sciences religieuses 64: 53-71.

FUCHS, J. 1977. "Lehrfach Moraltheologie als "sacra doctrina"», dans In libertatem vocati estis. Miscellanea Bernhard Häring. Roma, Academia Alfonsiana, (Studia Moralia \# XV), 191-206.

FURST, W. 1990. "Einblick in die Situation von Kirche und Universität in der modernen Gesellschaft", dans Katholische Theologie heute. Eine Einführung in das Studium. (WOHLMUTH, J., hrsg.), Würzburg, Echter, 21-32.

GAUTHIER, P. 1979. "La théologie à I'Université pour John Henry Newman", Bulletin de littérature ecclésiastique 80: 29-48.

HECKEL, M. 1986. Die theologischen Fakultäten im weltlichen Verfassungsstaat. Tübingen, J.C.B. Mohr (Paul Siebeck), (Jus Ecclesiasticum \#31), $416 \mathrm{p}$.

HOLLERBACH, A. 1982. "Die Theologischen Fakultäten und ihr Lehrpersonal im Beziehungsgefüge von Staat und Kirche", dans Theologie in der Universität. Münster, Aschendorff, (Essener Gespräche zum Thema Staat und Kirche \#16), 69-102 (Discussion: 103-170).

JONGH, H. de. 1980. L'ancienne faculté de théologie de Louvain au premier siècle de son existence (1432-1540). Ses débuts, son organisation, son enseignement, sa lutte contre Érasme et Luther. Uthrecht, Hes Publishers, 268 p., + 89*p. + XLVIIp. (Réimpression de l'édition Louvain, 1911)

JORDAN, M.D. 1988. "On Defending Catholic Higher Education in America", dans Readings in Moral Theology. No 6: Dissent in the Church. (CURRAN, C.E. and R.A. McCORMICK., éd.), New-YorkMahwah, Paulist Press, 290-303.

KOPETZKY, B. 1970. "Les facultés de théologie entre hier et demain", Concilium 57: 123-136.

KRIELE, M. 1990. "Aktuelle Probleme des Verhältnisses von Kirche und Staat", Internationale katholische Zeitschrift 19: 541-555. 
LADRIËRE, J. 1990. "L'université catholique et l'intelligence de la foi", dans Défis orésents et à venir de l'université catholique. Actes du colloque international du centenaire de l'Université Saint-Paul, 28-31 août 1989. Ottawa, Université Saint-Paul, 103-121.

LINK. C. 1988. "Der Rechtsstatus der Theologischen Fakultäten", Theologische Rundschau 53: 405-416.

LORENZ, H. 1990. "Erfahrungen von Pfarrern im Kontaktstudium», Pastoraltheologie 79: 326-333.

MEHL, R. 1988. "Légitimité de la présence d'une faculté de théologie au sein de l'Université", Revue d'histoire et de philosophie religieuses 68: 133-143.

MULERT, H. 1930. Evangelische Kirchen und theologische Fakultäten. Tübingen, J.C.B. Mohr (Paul Siebeck), (Sammlung gemeinverständlicher Vorträge und Schriften aus dem Gebiet der Theologie und Religionsgeschichte \#144), $40 \mathrm{p}$.

MULLER, H.M. 1990. "Kirchliche Praxis als Zielpunkt der Universitätstheologie", Pastoraltheologie 79: 317-326.

ORSY, L. 1987. The Church: Learning and Teaching. MagisteriumAssent-Dissent-Academic Freedom. Wilmington, Michael Glazier, $172 \mathrm{p}$.

PRESTON, R.H. 1981. "The Faculty of Theology in the University of Manchester: the First Seventy-Five Years", Bulletin of the John Bylands University Library of Manchester 63: 463-484.

PUZA, R. 1991. "Staatskirchenrechtliche und kirchenrechtliche Stellung und Aufgaben der Theologischen Fakultäten", Theologische Quartalschrift 171: 108-114.

RAHNER, K. 1983. "Theologie heute", dans RAHNER, K. Theologische Schriften. Band 15. Zürich-Einsiedeln-Köln, Benziger Verlag, $63-75$.

RATZINGER, J. 1981. "Théologie et politique ecclésiale", Communio 6: $29-40$.

RUH, U. 1986. «Theologie an der Universität. Situation und Probleme der theologischen Fakultäten", Herder Korrespondenz 40: 535-540.

SCHÄFER, R. 1970. "Die theologische Fakultät - ein staats - kirchliches Relikt", Club Voltaire 4: 286-298.

SCHILLEBEECKX, E. 1967. "La signification de I'Université catholique pour le monde et l'Église", dans SCHILLEBEECKX, E. Approches théologiques, III. Le monde et l'Église. Bruxelles, CEP, 312-325. 
Jean-Claude Petit La théologie dans l'université: un défi...

SCHMITZ, H. 1987. "Hochschulplanung nach Kirchlichen Recht", Theologie und Glaube 77: $347-360$.

SCHMITZ, H. 1987. "Kirchliches Recht für staatliche KatholischTheologische Fakultäten. Akkomodation kirchlichen Hochschulrechts an die deutschen Verhältnisse", Theologische Quartalschrift 167: 25-40.

SHAW, D.W.D. 1988. "Theology in the University. A Contemporary Scottish Perspective", Scottish Journal of Theology 41: 217231.

TRIPOLE, M.R. 1979. "Why teach Theology to a Catholic College Student Today?", Horizons 6: 265-276.

TURNER, H.W. 1967. "Theology and University. From an African Perspective", Zeitschrift fur Religions - und Geistesgeschichte 19: 114-126.

URRUTIA, F.J. 1989. “Ecclesiastical Universities and Faculties. (Canons 815-821)", Studia Cononica 23: 459-469.

VALCKE, L. 1984. "Université, théologie et virage technologique", L'analyste 7: 42-46.

WALF, K. 1989. "La relation des facultés de théologie catholique avec l'État et la Société: le cas de la République fédérale d'Allemagne", Studia canonica 23: 101-118. 